\title{
Association between the stress fracture and bone metabolism/quality markers in lacrosse players
}

This article was published in the following Dove Press journal:

Open Access Journal of Sports Medicine

23 July 2012

Number of times this article has been viewed

\section{Kenta Wakamatsu' \\ Keishoku Sakuraba' \\ Yoshio Suzuki \\ Asako Maruyama ${ }^{2}$ \\ Yosuke Tsuchiya ${ }^{3}$ \\ Jiro Shikakura ${ }^{2}$ \\ Eisuke Ochi ${ }^{3}$}

'Department of Sports Medicine, Graduate School of Medicine, Juntendo University, Tokyo, Japan; ${ }^{2}$ School of Health and Sports Science, Juntendo University, Chiba, Japan; ${ }^{3}$ Laboratory of Health and Sports Sciences, Meiji Gakuin University, Kanagawa, Japan
Correspondence: Eisuke Ochi Meiji Gakuin University, Laboratory of Health and Sports Sciences, I5I 8 Kamikurata-cho, Totsuka-ku, Yokohama, Kanagawa 244-8539, Japan

Tel +81458632144

Fax +8I458632I44

Email ochi@gen.meijigakuin.ac.jp
Background: Overuse injury including stress fracture is a serious problem for athletes. Recently, the importance of bone metabolism and quality as factors preventing overuse injury has been increasingly recognized. Hence, we hypothesized that markers of bone metabolism and quality are related to overuse injuries.

Methods: The subjects, which were elite university lacrosse players (male, $\mathrm{n}=35$; age, $19.8 \pm 1.1$; female, $\mathrm{n}=49$; age, $20.0 \pm 1.0$ ), were divided into a stress fracture group and a control group. We measured the subjects' physical characteristics (height, weight, body mass index, and body fat) and bone architecture was evaluated using quantitative ultrasound. Bone alkaline phosphatase, N-telopeptide cross-link of type I collagen, tartrate-resistant acid phosphatase 5b (TRAP-5b), homocysteine, and pentosidine were measured from blood samples obtained from all subjects.

Results: No significant difference was observed between groups with respect to height, weight, body mass index, and body fat, as well as quantitative ultrasound. Further, there were no significant differences in the levels of bone alkaline phosphatase, N-telopeptide cross-link of type I collagen, or TRAP-5b between stress fracture and control groups in all subjects and in male subjects. However, a significant increase in TRAP-5b level was observed in the stress fracture group compared with the control in the female subjects $(409.9 \pm 209.3$ and $318.6 \pm 81.6 \mathrm{mU} / \mathrm{dL}$, respectively; $P<0.05$ ). Homocysteine and pentosidine did not differ between groups.

Conclusion: These results suggest that osteoclast activity of female athletes with stress fractures may be enhanced by TRAP-5b.

Keywords: overuse injury, stress fracture, TRAP-5b

\section{Introduction}

Bone strength primarily reflects the integration of bone mineral density (BMD) and bone quality. ${ }^{1}$ Bone quality refers to architecture, turnover, metabolism, damage accumulation (eg, microfractures), and mineralization of bone. ${ }^{1}$ Overuse injuries, including stress fracture, are a serious problem for athletes, and it has been revealed that female athletes with amenorrhea have a high risk of stress fractures. ${ }^{2-4}$ Although previous studies have focused on bone mineral density and bone quality, the behavior of bone metabolism markers is still controversial. ${ }^{5}$

Bone metabolism markers frequently measured in previous studies include bone alkaline phosphatase (BAP) and N-telopeptide cross-link of type I collagen (NTx). ${ }^{6,7}$ However, these parameters are influenced by exercise, food, and renal function and show intra- and inter-day variations, which is problematic. ${ }^{7,8}$ Bone-specific tartrate-resistant acid phosphatase $5 \mathrm{~b}$ (TRAP-5b) has recently been reported as a 
new bone resorption marker that shows small intra- and inter-day variations. ${ }^{9-13}$ Most studies examining TRAP-5b have focused on osteoporosis patients and postmenopausal females; ${ }^{9-11}$ there have been no studies involving athletes. In addition to the markers described above, it has been shown that collagen cross-links are important factors influencing bone quality. ${ }^{1,14-16}$ Collagen cross-links can be divided into enzymatic cross-links (lysyl oxidase-mediated cross-links) and nonenzymatic cross-links (advanced glycation end products; AGEs) types. ${ }^{14}$ In contrast to the positive effects conferred by enzymatic cross-links, nonenzymatic cross-links have detrimental effects on the mechanical and biological functions of bone. ${ }^{17}$ Homocysteine is known to inhibit enzymatic cross-link formation via reduction of gene expression and enzymatic activity of lysyl oxidase. ${ }^{18,19}$ Pentosidine is an AGE formed by nonenzymatic glycation or oxidation reactions resulting in tissue maturation..$^{20,21}$ Although previous reports have shown that homocysteine and pentosidine are significantly related to daily life habits, ${ }^{14,17}$ the role of these factors with exercise intervention remains unknown.

In this study, we investigated the relationship between past stress fractures, quantitative ultrasound (QUS), and bone metabolism and quality markers (BAP, NTx, TRAP-5b, homocysteine, and pentosidine). The study aimed to determine markers that should be monitored to help prevent overuse injury in university lacrosse players.

\section{Methods}

\section{Subjects}

The subjects included 84 elite university lacrosse players (Table 1). Before the study, we questioned subjects regarding past medical history of stress fracture, which was diagnosed by experienced orthopedic surgeons. All subjects were instructed to base their answers on their past status in our original questionnaire. Based on this investigation, subjects were divided into groups with ( 5 males and 7 females) and without (30 males and 42 females) stress fracture. The average number of months subjects were injured was $14.6 \pm 10.7$ months (from 2 to 36 months). In addition, skeletal sites were classified into three groups: (1) metatarsal bone ( 6 cases, $50 \%$ ), (2) tibial bone ( 4 cases, 33\%), (3) others ( 2 cases, 17\%). This study was conducted with the approval by the Ethics Committee of Nippon Sports Science University (ID: 21-15). The objective, content, and points of caution were explained to the subjects, and informed consent was obtained from all subjects before the study.

\section{Body composition measurement}

Body composition was measured using the Tanita MC-190 body composition analyzer (Tanita Corp, Tokyo, Japan) with correction for light indoor clothing. ${ }^{22}$ The measurement procedure required the subject to stand barefoot on the analyzer and to hold a pair of handgrips (one in each hand). The device uses multiple-frequency $(5 \mathrm{kHz}, 50 \mathrm{kHz}$, $250 \mathrm{kHz}$, and $500 \mathrm{kHz}$ ) bioelectrical impedance analysis technology and has 8 tactile electrodes: 2 are in contact with the palm and thumb of each hand and 2 are in contact with the anterior and posterior aspect of the sole of each foot.

\section{Quantitative ultrasonic measurement}

Using a QUS device (AOS-100; Aloka Co, Tokyo, Japan), the osteo-sono assessment index of the right calcaneous was measured as an indicator of bone strength. During the measurement, two transducers were fixed coaxially on both sides of the calcaneus by a caliper and were coupled to the skin using coupling gel. Validation of this method and its high reproducibility compared with parameters obtained from the speed of sound (SOS) and broadband ultrasound attenuation has been reported in a previous study. ${ }^{23}$ The SOS and the transmission index (TI) of the right heel were measured and OSI was calculated using the following formula: $\mathrm{OSI}=\mathrm{TI} \times \mathrm{SOS} \times \mathrm{SOS}(\mathrm{CV} 1.31,10$ repeats $)$.

Table I Physical characteristics of subjects

\begin{tabular}{lccccc}
\hline & Age (year) & Height $(\mathbf{c m})$ & Weight $(\mathbf{k g})$ & BMI & Body fat $(\%)$ \\
\hline Male & & & & & \\
$\quad$ All subjects $(\mathrm{n}=35)$ & $19.8 \pm 1.1$ & $172.0 \pm 3.9$ & $65.9 \pm 4.6$ & $22.3 \pm 1.4$ & $12.0 \pm 1.9$ \\
$\quad$ Stress fracture group $(\mathrm{n}=5)$ & $19.6 \pm 0.5$ & $171.4 \pm 5.5$ & $64.0 \pm 7.3$ & $21.8 \pm 2.1$ & $12.0 \pm 0.9$ \\
$\quad$ Control group $(\mathrm{n}=30)$ & $19.9 \pm 1.1$ & $172.0 \pm 3.6$ & $66.2 \pm 4.0$ & $22.4 \pm 1.3$ & $12.0 \pm 2.0$ \\
Female & & & & & \\
$\quad$ All subjects $(\mathrm{n}=49)$ & $20.0 \pm 1.0$ & $160.4 \pm 5.8$ & $56.7 \pm 5.8$ & $22.0 \pm 2.0$ & $25.4 \pm 4.3$ \\
$\quad$ Stress fracture group $(\mathrm{n}=7)$ & $20.6 \pm 1.4$ & $160.0 \pm 3.7$ & $54.5 \pm 5.2$ & $21.3 \pm 1.8$ & $24.3 \pm 3.6$ \\
Control group $(\mathrm{n}=42)$ & $19.9 \pm 0.9$ & $160.4 \pm 6.1$ & $57.1 \pm 5.8$ & $22.1 \pm 2.1$ & $25.5 \pm 4.4$ \\
\hline
\end{tabular}

Note: Mean \pm SD

Abbreviation: BMI, body mass index. 


\section{Bone metabolism and quality markers}

Prior to blood sampling, the subjects refrained from food ingestion for 12 hours and exercise for 24 hours. BAP, as an indicator of bone formation, and NTx and TRAP-5b, as indicators of bone resorption, were measured to characterize bone metabolism. Homocysteine and pentosidine were also measured as bone quality markers. To minimize intra-day variations in these bone metabolism markers, blood was collected at the same time (morning) and off-season of competitions. Blood markers were measured at the SRL laboratory (Tokyo, Japan) according to a method reported previously. ${ }^{24,25}$

\section{Statistical analysis}

All values are presented as the mean \pm standard deviation. Because of the small sample size and nonnormality of the data in this cross-sectional trial, exact nonparametric tests were used. The exact Mann-Whitney $U$-test was used to compare changes between the two groups. SPSS software (version 18.0 for Windows; SPSS Inc, Chicago, IL) was used to analyze the significance of differences. The significance level was set at 0.05 for all tests.

\section{Results}

\section{Physical characteristics}

The physical characteristics of the subjects are shown in Table 1. No significant difference was observed between the stress fracture and control groups with respect to age (Table 1; male stress fracture, $19.6 \pm 0.5$; male control, $19.9 \pm 1.1$; female stress fracture, $20.6 \pm 1.4$; female control, 19.9 \pm 0.9 ). Further, there were no significant differences between groups with respect to height, weight, body mass index, or body fat (Table 2).

\section{Quantitative ultrasonic measurement}

The results of QUS measurements are shown in Table 2. The QUS data did not differ between subjects (stress fracture, $3.50 \pm 0.34$; control, $3.46 \pm 0.42$ ), the male group (stress fracture; $3.50 \pm 0.38$, control; $3.50 \pm 0.36$ ), or the female group (stress fracture, $3.49 \pm 0.33$; control, $3.43 \pm 0.45$ ).

\section{Bone metabolism and quality markers}

No significant differences were observed in the levels of BAP, NTx, and TRAP-5b between the stress fracture and control groups for all subjects (Table 2). In male players, no significant differences were observed between the stress fracture and control groups for BAP, NTx, and TRAP-5b (Table 2). Similarly, in female players, no significant

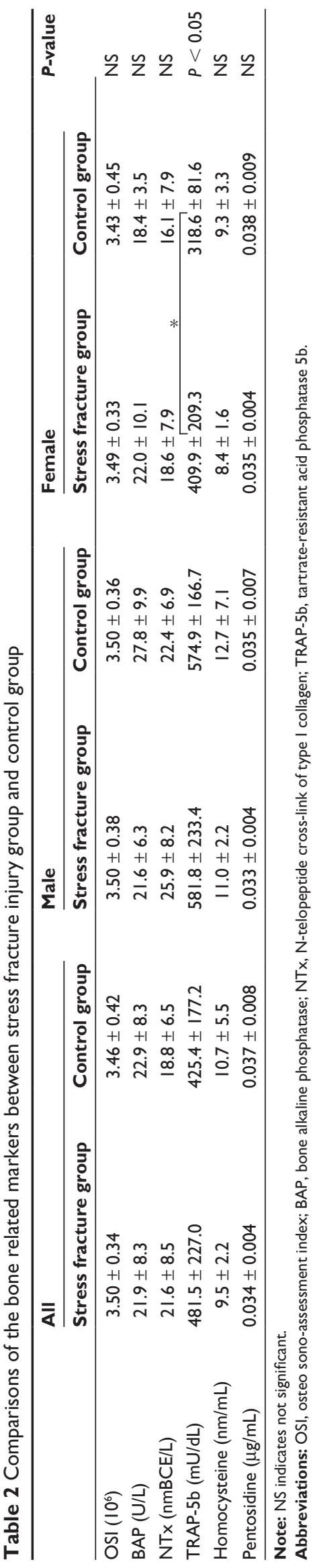


differences were observed in the levels of BAP or NTx (Table 2). However, in the female players, TRAP-5b levels were $409.9 \pm 209.3$ and $318.6 \pm 81.6 \mathrm{mU} / \mathrm{dL}$ in the stress fracture and control groups, respectively. A significant increase in TRAP-5b levels was observed in the stress fracture group $(P<0.05$, Table 2$)$.

Homocysteine and pentosidine were analyzed in all subjects (Table 2). Homocysteine did not differ between groups (all stress fracture subjects, $9.5 \pm 2.2$; all control subjects, $10.7 \pm 5.5$; male stress fracture, $11.0 \pm 2.2$; male control, $12.7 \pm 7.1$; female stress fracture, $8.4 \pm 1.6$; female control, $9.3 \pm 3.3$ ). Further, no significant difference was observed between the stress fracture and control groups with respect to pentosidine.

\section{Discussion}

Because bone quality is an important factor for maintaining bone strength, ${ }^{1,14-16}$ we evaluated several bone metabolism markers as well as bone in overuse injury. It has recently been proposed that $70 \%$ of bone strength depends on bone mineral density (BMD), and the remaining $30 \%$ on bone quality. ${ }^{1}$ While there are various indicators of $\mathrm{BMD}$, none reflect the true condition of bone at the time of measurement. Thus, we measured blood NTx, BAP, TRAP-5b, homocysteine, and pentosidine levels and performed QUS. Our results show that significantly higher TRAP-5b levels were observed only in female players in the stress fracture group (Table 2). In contrast, it has been shown that TRAP in male and female patients taking oral anticoagulants significantly increases with a concomitant decrease in QUS. ${ }^{26}$ Although the relationship between TRAP-5b and QUS requires further clarification, these results suggest that there is an association between TRAP-5b and bone strength and/or overuse injuries in female athletes.

Previous studies have shown that menstruation status, vitamin $\mathrm{D}$, and calcium play critical roles in maintaining bone strength. 5,27,28 Particularly, gender differences in hormones are considered to be the source of the significant difference in TRAP-5b levels observed only in female players. Estrogen deficiency is known to promote bone resorption, particularly in postmenopausal females. ${ }^{29-31}$ Because enhanced bone resorption reduces bone mass, it is important to investigate estrogen levels in relation to injury in females. Previous studies have reported that estrogen maintains bone mass and that BMD was low in athletes with exercise amenorrhea. ${ }^{5,30,32}$ Hence, the association between menstruation status, including estrogen and TRAP-5b, in stress fracture patients is an important area for future studies.
In our study, homocysteine and pentosidine, markers associated with collagen cross-links, did not differ significantly between stress fracture and control groups (Table 2). AGEs, including pentosidine, are formed by oxidation reactions that result in tissue maturation. ${ }^{33}$ It is commonly understood that oxidative stress is caused by intense exercise. ${ }^{34}$ In our study, these makers were not measured during a period of intense training; thus, the absence of significant differences may be in part attributable to the low oxidative stress period. Further investigation, including comparisons between nontraining and intense training periods, may be necessary.

In conclusion, our results demonstrate that TRAP-5b may be a useful bone metabolism marker for monitoring bone status in female athletes. Although further investigation of gender differences, estrogen levels, nutrition status, and oxidative stress are necessary to elucidate the mechanism of overuse injuries, the osteoclast activity of female athletes with stress fracture may be enhanced by TRAP-5b.

\section{Disclosure}

The authors did not receive and will not receive any benefits and funding from any commercial party related directly or indirectly to the subject of this article.

\section{References}

1. NIH Consensus Development Panel on Osteoporosis Prevention, Diagnosis, and Therapy. Osteoporosis prevention, diagnosis, and therapy. JAMA. 2001;285(6):785-795.

2. Louis O, Demeirleir K, Kalender W, et al. Low vertebral bone density values in young non-elite female runners. Int J Sports Med. 1991;12(2): 214-217.

3. Marcus R, Cann C, Madvig P, et al. Menstrual function and bone mass in elite women distance runners. Ann Intern Med. 1985;102(2): $158-163$.

4. Anahid TC. Nutrition for Sport and Exercise: 2nd ed, Jacqueline R. Berning and Suzanne Nelson Steen. Gaithersburg, MD: Aspen Publishers. J Am Coll Nutr April. 2000;19:285.

5. Ducher G, Turner AI, Kukuljan S, et al. Obstacles in the optimization of bone health outcomes in the female athlete triad. Sports Med. 2011;41(7):587-607.

6. Banfi G, Lombardi G, Colombini A, Lippi G. Bone metabolism markers in sports medicine. Sports Med. 2010;40(8):697-714.

7. Delmas PD, Eastell R, Garnero P, Seibel MJ, Stepan J. The use of biochemical markers of bone turnover in osteoporosis. Committee of Scientific Advisors of the International Osteoporosis Foundation. Osteoporos Int. 2000;11(Suppl 6):S2-S17.

8. Ju HS, Leung S, Brown B, et al. Comparison of analytical performance and biological variability of three bone resorption assays. Clin Chem. 1997;43(9):1570-1576.

9. Alatalo SL, Halleen JM, Hentunen TA, Mönkkönen J, Väänänen HK. Rapid screening method for osteoclast differentiation in vitro that measures tartrate-resistant acid phosphatase $5 \mathrm{~b}$ activity secreted into the culture medium. Clin Chem. 2000;46(11):1751-1754.

10. Halleen JM. Tartrate-resistant acid phosphatase $5 \mathrm{~B}$ is a specific and sensitive marker of bone resorption. Anticancer Res. 2003;23(2A): 1027-1030. 
11. Ohashi T, Igarashi Y, Mochizuki Y, et al. Development of a novel fragments absorbed immunocapture enzyme assay system for tartrateresistant acid phosphatase 5b. Clin Chim Acta. 2007;376(1-2): 205-212.

12. Shiraki M, Kuroda T, Shiraki Y, Tanaka S, Higuchi T, Saito M. Urinary pentosidine and plasma homocysteine levels at baseline predict future fractures in osteoporosis patients under bisphosphonate treatment. J Bone Miner Metab. 2011;29(1):62-70.

13. Yamada S, Inaba M, Kurajoh M, et al. Utility of serum tartrate-resistant acid phosphatase (TRACP5b) as a bone resorption marker in patients with chronic kidney disease: independence from renal dysfunction. Clin Endocrinol (Oxf). 2008;69(2):189-196.

14. Saito M, Fujii K, Marumo K. Degree of mineralization-related collagen crosslinking in the femoral neck cancellous bone in case of hip fracture and controls. Calcif Tissue Int. 2006;79(3):160-168.

15. Saito M, Marumo K, Soshi S, Kida Y, Ushiku C, Shinohara A. Raloxifene ameliorates detrimental enzymatic and nonenzymatic collagen cross-links and bone strength in rabbits with hyperhomocysteinemia. Osteoporos Int. 2010;21(4):655-666.

16. Shidara K, Inada M, Okuno S, et al. Serum levels of TRAP-5b, a new bone resorption marker unaffected by renal dysfunction, as a useful marker of cortical bone loss in hemodialysis patients. Calcif Tissue Int. 2008;82(4):278-287.

17. Saito M, Marumo K. Collagen cross-links as a determinant of bone quality: a possible explanation for bone fragility in aging, osteoporosis, and diabetes mellitus. Osteoporos Int. 2010;21(2):195-214.

18. Khan M, Yamauchi M, Srisawasdi S, et al. Homocysteine decreases chondrocyte-mediated matrix mineralization in differentiating chick limb-bud mesenchymal cell micro-mass cultures. Bone. 2001;28(4): 387-398.

19. Raposo B, Rodriquez C, Martinez-Gonzalez J, Badimon L. High levels of homocysteine inhibit lysyl oxidase(LOX) and downregulate LOX expression in vascular endothelial cells. Atherosclerosis. 2004; 177(1):1-8.

20. Paul RG, Bailey AJ. Glycation of collagen: the basis of its central role in the late complications of ageing and diabetes. Int J Biochem Cell Biol. 1996;28(12):1297-1310.

21. Sell DR, Monnier VM. Structure elucidation of a senescence cross-link from human extracellular matrix. Implication of pentoses in the aging process. J Biol Chem. 1989;264(36):21597-21602.
22. Sugawara N, Yasui-Furukori N, Tsuchimine S, et al. Body composition in patients with schizophrenia: comparison with healthy controls. Ann Gen Psychiatry. 2012;11(1):11.

23. Tsuda-Futami E, Hans D, Njeh CF, et al. An evaluation of a new gelcoupled ultrasound device for the quantitative assessment of bone. $\mathrm{Br}$ J Radiol. 1999;72(859):691-700.

24. Lombardi G, Colombini A, Freschi M, Tavana R, Banfi G. Seasonal variation of bone turnover markers in top-level female skiers. Eur $J$ Appl Physiol. 2011;111(3):433-440.

25. Shiraki M, Urano T, Kuroda T, et al. The synergistic effect of bone mineral density and methylenetetrahydrofolate reductase (MTHFR) polymorphism(C677T) on fractures. J Bone Miner Metab. 2008;26(6): 595-602.

26. Rey-Sanchez P, Lavado-Garcia JM, Canal-Macias ML, RodriquezDominquez MT, Bote-Mohedano JL, Pedrera-Zamorano JD. Ultrasound bone mass in patients undergoing chronic therapy with oral anticoagulants. J Bone Miner Metab. 2011;29(5):546-551.

27. Turner AG, Anderson PH, Morris HA. Vitamin D and bone health. Scand J Clin Lab Invest. 2012;72(Suppl 243):65-72.

28. Ondrak KS, Morgan DW. Physical Activity, Calcium intake and bone health in children and adolescents. Sports Med. 2007;37(7):587-600.

29. Kohrt WM, Snead DB, Slatopolsky E, Birge SJ Jr. Additive effects of weight-bearing exercise and estrogen on bone mineral density in older women. J Bone Miner Res. 1995;10(9):1303-1311.

30. Lee KC, Lanyon LE. Mechanical loading influences bone mass through estrogen receptor alpha. Exerc Sport Sci Rev. 2004;32(2):64-68.

31. Riggs BL. Endocrine causes of age-related bone loss and osteoporosis. Novartis Found Symp. 2002;242:247-259.

32. Mesaki N, Motobu M, Sasaki J. Decreased bone mineral density in athletic amenorrhea. Jpn J Fertil Steril. 1994;39(4):11-16.

33. McCarthy AD, Etcheverry SB, Bruzzone L, Lettieri G, Barrio DA, Cortizo AM. Non-enzymatic glycosylation of a type I collagen matrix: effects on osteoblastic development and oxidative stress. BMC Cell Biol. 2001;2:16.

34. Packer L. Oxidants, antioxidant nutrients and the athlete. J Sports Sci. 1997;15(3):353-363.
Open Access Journal of Sports Medicine

\section{Publish your work in this journal}

Open Access Journal of Sports Medicine is an international, peer-reviewed, open access journal publishing original research, reports, reviews and commentaries on all areas of sports medicine. The manuscript management system is completely online and includes a very quick and fair peer-review system.

\section{Dovepress}

Visit http://www.dovepress.com/testimonials.php to read real quotes from published authors. 